UTHEP-339, August 1996

\title{
Tensor Charge of the Nucleon in Lattice QCD
}

\author{
S. Aoki, ${ }^{1}$ M. Doui1 ${ }^{1}$, T. Tatsuda ${ }^{1}$ and Y. Kuramashi ${ }^{2}$ \\ ${ }^{1}$ Institute of Physics, University of Tsukuba, Tsukuba, Ibaraki 305, Japan \\ ${ }^{2}$ National Laboratory for High Energy Physics (KEK), Tsukuba, Ibaraki 305, Japan
}

\begin{abstract}
First results of lattice QCD simulation on the nucleon tensor-charge $\delta q$ are presented. From the quenched QCD simulations with the Wilson quark action at $\beta=5.7$ on a $16^{3} \times 20$ lattice and on a $12^{3} \times 20$ lattice, we find (i) the connected part $\delta q_{\text {con }}$. is determined with small statistical error, (ii) the disconnected part $\delta q_{d i s}$, which violates the OZI rule, is consistent with zero, and (iii) the flavor-singlet tensor charge $\delta \Sigma\left(\mu^{2}=2 \mathrm{GeV}^{2}\right)=\delta u+\delta d+\delta s$ takes $0.562(88)$ at $\beta=5.7$, which is in contrast with the flavor-singlet axial charge $\Delta \Sigma=0.1-0.3$.
\end{abstract}

PACS numbers: 13.88.+e, 12.38.Gc, 14.20.Dh

Typeset using REVTEX 
The parton structure of the nucleon at the twist two level is known to be characterized by three structure functions $f_{1}(x, \mu), g_{1}(x, \mu)$ and $h_{1}(x, \mu)$ with $x$ being the Bjorken variable and $\mu$ being the renormalization scale (see, [1]). $f_{1}$ and $g_{1}$, which represent the quark-momentum distribution and the quark-spin distribution in the nucleon respectively, can be measured by the deep inelastic lepton-hadron scattering. On the other hand, $h_{1}(x)$, which represents the quark-transversity distribution, could be measured in the polarized Drell-Yan processes, since it is related to the matrix element of the chiral-odd quark operator. Such experiment is planned using the relativistic heavy ion collider (RHIC) at BNL. Therefore, theoretical prediction of $h_{1}$ is of great importance. Also, whether there is a large OZI violation in the first moment of $h_{1}(x)$ has particular interest since the first moment of $g_{1}(x)$ and that of the twist three structure function $e(x)$ have been argued to have large OZI violation from experimental and/or theoretical point of view [2, 3.4. In this paper, we will report first results on the first moment of $h_{1}(x)$ in lattice QCD simulations [5].

The tensor charge of the nucleon is defined as $\delta q(\mu)=\int_{0}^{1}\left[h_{1}(x, \mu)-\bar{h}_{1}(x, \mu)\right] d x$, where $h_{1}(x)$ and $\bar{h}_{1}(x)$ are the quark and anti-quark distributions respectively. One can also write $\delta q$ as a matrix element of the tensor operator

$$
\left\langle p s\left|\bar{q} i \sigma_{\mu \nu} \gamma_{5} q\right| p s\right\rangle=2\left(s_{\mu} p_{\nu}-s_{\nu} p_{\mu}\right) \delta q,
$$

where $p_{\mu}$ is the nucleon's four momentum, and $s_{\mu}$ is the nucleon's covariant spin-vector normalized as $s_{\mu}^{2}=-1$ [6]. In the nucleon light-cone frame, $\delta q$ is interpreted as the "transversity" of quarks in a transversely polarized nucleon [1]. On the other hand, in the nucleon rest frame with $\mu=0$ and $\nu=3, \delta q \sim\left\langle p s\left|\bar{q}\left(\begin{array}{cc}\sigma_{3} & 0 \\ 0 & \sigma_{3}\end{array}\right) q\right| p s\right\rangle$. This implies that $\delta q$ gives an independent information on the spin structure of the nucleon from the axial charge $\Delta q \sim\left\langle p s\left|\bar{q} \gamma_{3} \gamma_{5} q\right| p s\right\rangle \sim\left\langle p s\left|\bar{q}\left(\begin{array}{cc}\sigma_{3} & 0 \\ 0 & -\sigma_{3}\end{array}\right) q\right| p s\right\rangle$.

As for $\delta q$, approximate but model-independent inequalities such as $|\delta u|<3 / 2$ and $|\delta d|<1 / 3$ are known [7]. The non-relativistic quark model predicts $\delta u=\Delta u=4 / 3$ and $\delta d=\Delta d=-1 / 3$, while the relativistic quark wave functions with non-vanishing lower 
components lead to $\delta u=1.17$ and $\delta d=-0.29$ together with the inequality $|\delta q|>|\Delta q|[8]$. There also exist estimates of $\delta q$ using QCD sum rules [8,9] and a chiral quark model [10], but the uncertainties of the results are either large or uncontrollable.

In lattice QCD simulations, one can treat both the connected (OZI preserviong) and disconnected (OZI violating) contributions even in the quenched approximation as has been demonstrated in [11,12]. Also, one can estimate systematic errors due to the lattice approximation by combining simulations with different lattice spacing and/or lattice size.

On the lattice, the matrix element of the tensor operator $O(t, \mathbf{x})=\bar{q}\left(\begin{array}{cc}\sigma_{3} & 0 \\ 0 & \sigma_{3}\end{array}\right) q$ is extracted as

$$
\begin{aligned}
R(t) & \equiv \frac{\sum_{s= \pm} s\left\langle N_{s}(t) \sum_{t^{\prime} \neq 0, x} O\left(t^{\prime}, \mathbf{x}\right) \bar{N}_{s}(0)\right\rangle}{\sum_{s= \pm}\left\langle N_{s}(t) \bar{N}_{s}(0)\right\rangle} \\
& \rightarrow \text { const. }+Z_{T}^{-1} \delta q t \quad \text { (large t), }
\end{aligned}
$$

where $Z_{T}$ is the lattice renormalization factor for the tensor operator and $N(t)$ is the nucleon operator projected to zero momentum.

Following the works in ref. [11] where similar simulations have been done, we have taken into account the points (i) (iv) below.

(i) We adopt the Wilson quark action and take $N=\left(q^{t} C^{-1} \gamma_{5} q\right) q$ as a nucleon interpolating field. To enhance the nucleon signal, we use a wall source at initial time slice $t=0$ with the Coulomb gauge fixing at $t=0$. Other time slices are not gauge-fixed.

(ii) To avoid the contribution of the negative-parity nucleon propagating backward in time, the Dirichlet boundary condition for the quark propagator in the temporal direction is adopted.

(iii) The connected amplitude is calculated by the conventional source method [13]. To obtain the disconnected amplitude, the quark propagator with space-time unit source without gauge fixing is adopted. By this procedure, only the gauge invariant closed-loop is automatically selected after the average over the gauge configurations $[11]$. Note that $t^{\prime}=0$ must be excluded in the sum in eq.(2) to make the procedure consistent. 
(iv) To compare the matrix element on the lattice with that in the $\overline{M S}$ scheme, we employ the tadpole-improved renormalization factor $Z_{T}(\mu a)$. At $\mu=1 / a$ with $a$ being the lattice spacing, $Z_{T}$ in the Wilson quark action reads 14

$$
Z_{T}=\left[1-\left(\frac{2}{3 \pi} \ln (\mu a)+0.44\right) \alpha_{\overline{M S}}(1 / a)\right]\left(1-\frac{3 K}{4 K_{c}}\right)
$$

where $K$ is the hopping parameter. The strong coupling constant $\alpha \overline{M S}(1 / a)$ is evolved down by the 2-loop renormalization group from $\alpha \overline{M S}(\pi / a)$ given by $\alpha \overline{M S}(\pi / a)^{-1}=P \alpha_{0}^{-1}+0.30928$. Here $P$ is the plaquette expectation value and $\alpha_{0}$ is the bare lattice coupling constant [15].

We carry out the quenched QCD simulation with the Wilson quark action at $\beta=5.7$ on a $16^{3} \times 20$ lattice. In order to estimate the finite size effect, simulations at $\beta=5.7$ on a $12^{3} \times 20$ lattice are also performed. Simulation parameters and some of the measured quantities are summarized in Table @. Gauge configurations, which are generated with a single plaquette action separated by 1000 pseudo heat bath sweeps, are analyzed for three hopping parameters. The $u$ and $d$ quarks are assumed to be degenerate with $K_{u}=K_{d}=K_{q}$, while the strange quark is assigned a different hopping parameter $K_{s}$. The statistical errors of the data points are estimated by the single elimination jackknife procedure, and final fit of the hadron masses and $R(t)$ are done by the $\chi^{2}$-fitting. The lattice spacing $a$ is extracted from $m_{\rho} a$ in the chiral limit.

The connected and disconnected parts of $R(t)$ at $\beta=5.7$ on a $16^{3} \times 20$ lattice are shown in Fig. 1 at $K=0.164$. Clear linear behaviors are seen for the $\mathrm{u}$ and $\mathrm{d}$ connected contributions $\delta q_{\text {con. }}$ in the interval up to $t \sim 11$ even for the smallest quark mass $K=0.1665$. For $t>11$, errors grow rapidly. The disconnected contribution $\delta q_{d i s}$, are concentrating around zero with errors comparable to the signal below $t=11$. Since the disconnected part of $R(t)$ does not show clear signal of a linear slope and we find no other sensible criterion, we take the same interval to extract $\delta q_{d i s}$. in our linear fit. We adopt the same interval also for the fit of hadron masses. The linear fit of the connected part, the disconnected part and their sum are done separately. Our data on $\delta q$ is given in Table II.

For $\mathrm{u}$ and $\mathrm{d}$ quarks the fitted values are linearly extrapolated to the chiral limit $m_{q} a=$ 
$\left(1 / K_{q}-1 / K_{c}\right) / 2=0$. For the strange disconnected contribution, we first make linear interpolation to the physical strange quark mass $m_{s} a=\left(1 / K_{s}-1 / K_{c}\right) / 2$ at each fixed value of $K_{q}$ for nucleon, and then the results are again linearly extrapolated to the chiral limit $m_{q} a=\left(1 / K_{q}-1 / K_{c}\right) / 2=0$. The physical strange quark mass $m_{s} a$ is estimated by generalizing the relation $m_{\pi}^{2} a^{2}=A m_{q} a$, obtained from the hadron mass results, to $m_{K}^{2} a^{2}=A\left(m_{q} a+m_{s} a\right) / 2$ and using the experimental ratio $m_{K} / m_{\rho}=0.64$. The errors of the sum of $u, d$ and $s$ contributions are estimated by quadrature.

In Fig. 2, $\delta u_{c o n .}, \delta d_{c o n}$. and $\delta u_{d i s .}=\delta d_{d i s .}$ at $\beta=5.7$ on a $16^{3} \times 20$ lattice are shown as a function of $m_{q} a=\left(1 / K_{q}-1 / K_{c}\right) / 2$. The values linearly extrapolated down to the chiral limit are also shown with error bars.

Our final results extrapolated to the chiral limit at $\beta=5.7$ on a $16^{3} \times 20$ lattice are summarized in Table [II]. The results for $L=12(\beta=5.7)$, which are done to estimate the finite size effect, are also shown in the table. To compare our data on $\delta q$ with that of $\Delta q$, results of a previous simulation for $\Delta q$ in ref. [11] at $\beta=5.7$ on a $16^{3} \times 20$ lattice are presented.

From Table प] and 피, one finds the following features.

1. Both the connected and total tensor charges at $\beta=5.7$ on a $16^{3} \times 20$ lattice satisfy the inequalities $|\delta u|>|\Delta u|$ and $|\delta d|<|\Delta d|$. This is different from the prediction of naive quark models $|\delta q|>|\Delta q|$ mentioned before.

2. We did not see clear linear slope for the disconnected parts and we could not make definite conclusion of its precise value from our simulation. Nevertheless, rather small error bars $(\sim 0.05)$ for $\delta q_{d i s}$. in our main simulation $(L=16$ at $\beta=5.7)$ indicate that the OZI violation in $\delta q$ is small with a conservative upper bound $\left|\delta q_{d i s .}\right|<0.1$. Also, one notices that the disconnected part suffers a large finite volume effect. This is seen by comparing the data with smaller lattice sizes ( $L=12$ at $\beta=5.7$ ). The smallness of $\delta q_{d i s}$. could be related to the $\mathrm{C}$ (charge conjugation)-odd and chiral-odd nature of the tensor operator $\bar{q} \sigma_{\mu \nu} \gamma_{5} q$ [16].

3. Due to the small disconnected contributions, the flavor singlet tensor charge $\delta \Sigma=$ 
$\delta u+\delta d+\delta s$ is not much suppressed from its quark model value. This is in contrast to the flavor singlet axial charge $\Delta \Sigma$ which is known to be largely suppressed [2, [4].

We have also done the simulation at $\beta=6.0$ on a $16^{3} \times 20$ lattice $\left(a^{-1}=2.271(9) \mathrm{GeV}\right.$, $L a=1.39 \mathrm{fm}$ ) and obtained qualitatively the same results as above. In the future, sim-

ulations on a larger volume at $\beta=6.0$ should be done to extract a definite value of the connected and disconnected tensor charges in the continuum limit as well as to reduce the finite size errors.

\section{ACKNOWLEDGEMENTS}

The authors thank M. Okawa and A. Ukawa for helpful discussions and encouragements. Numerical calculation of this work was done Fujitsu VPP500 at Univ. of Tsukuba. This

work was supported in part by Univ. of Tsukuba Research Projects and in part by the Grants-in-Aid of the Japanese Ministry of Education, Science and Culture (No. 06102004). 


\section{REFERENCES}

* Present address: Research and Development Dep., Rikagaku Industrial Cooperation, Osaka 569-11, Japan.

[1] R. L. Jaffe and X. Ji, Nucl. Phys. B375, 527 (1992) and references therein.

[2] For the possibility of the large OZI violation in the axial vector channel and its relation to the nucleon spin problem, see G. Altarelli and G. Ridolfi, Nucl. Phys. B (Proc. Suppl.) 39B,C, 106 (1995).

[3] For the OZI violation in the scalar channel and the $\pi-N \sigma$-term, see T. Hatsuda and T. Kunihiro, Phys. Rep. 247, 221 (1994) and references therein.

[4] The current status of the lattice simulation in the flavor singlet channel is summarized in M. Okawa, Nucl. Phys. B (Proc. Suppl.) 47, 160 (1996).

[5] Preliminary report of the this study was presented at Australia-Japan workshop on Quarks, Hadrons and Nuclei (Nov. 15-24, 1995, Adelaide, Australia) and at RIKEN symposium on Spin Structure of the Nucleon (RIKEN, Japan, Dec. 18-19, 1995): report-no. Univ. of Tsukuba report UTHEP-337, hep-lat/9606006.

[6] Here we use Minkowski notation with the Bjorken-Drell convention.

[7] J. Soffer, Phys. Rev. Lett. 74, 1292 (1995).

[8] H. He and X. Ji, Phys. Rev. D52, 2960 (1995).

[9] B. L. Ioffe and A. Khodjamiraian, Phys. Rev. D51, 3373 (1995).

[10] H.-C. Kim, M. Polyakov and K. Goeke, Phys. Rev. D53, R4715 (1996); hep-ph/9604442.

[11] M. Fukugita, Y. Kuramashi, M. Okawa and A. Ukawa, Phys. Rev. D51 (1995) 5319; Phys. Rev. Lett. 75, 2092 (1995).

[12] S. J. Dong, J.-F. Lagae and K. F. Liu, Phys. Rev. Lett. 75, 2096 (1995). 
[13] C. Bernard et al., in Lattice Gauge Theory: A Challenge in Large-Scale Computing, edited by B. Bunk et al., (Plenum, New York, 1986); G. W. Kilcup et al., Phys. Lett. 164B, 347 (1985).

[14] G. P. Lepage and P. B. Mackenzie, Phys. Rev. D48, 2250 (1993).

[15] A.X.El-Khadra et al., Phys. Rev. Lett. 69, 729 (1992).

[16] X. Ji and J. Tang, Phys. Lett. B362, 182 (1995). 


\section{FIGURES}

FIG. 1. $R(t)$ as a function of $t$ at $K=0.164$ and $\beta=5.7$ on a $16^{3} \times 20$ lattice.

FIG. 2. Quark mass dependence of the tensor charges at $\beta=5.7$ on a $16^{3} \times 20$ lattice. Crosses with error bars denote the values extrapolated to the chiral limit. 


\section{TABLES}

\begin{tabular}{|c|ll|}
\hline \hline$\beta$ & 5.7 & \\
$L^{3} \times T$ & $16^{3} \times 20$ & $12^{3} \times 20$ \\
\hline$\#$ of conf. & 1053 & 306 \\
$K$ & $0.16,0.164,0.1665$ & $0.16,0.164,0.1665$ \\
fit range & $6 \leq t \leq 11$ & $5 \leq t \leq 10$ \\
\hline$K_{c}$ & $0.16925(3)$ & $0.16928(7)$ \\
$a^{-1}(\mathrm{GeV})$ & $1.418(9)$ & $1.452(25)$ \\
$L a(\mathrm{fm})$ & 2.23 & 1.63 \\
$\alpha_{M S}(1 / a)$ & 0.2158 & 0.2158 \\
\hline$m_{\rho} a$ & $0.5429(36)$ & $0.5303(92)$ \\
$m_{N} a$ & $0.8024(65)$ & $0.7985(144)$ \\
$m_{s} a$ & $0.0896(12)$ & $0.0857(30)$ \\
$A$ & $2.752(13)$ & $2.746(33)$ \\
\hline \hline
\end{tabular}

TABLE I. Summary of simulation parameters. 


\begin{tabular}{|lcccccc|}
\hline \hline \multicolumn{2}{|c}{$K_{q}$} & $\delta u_{\text {con. }}$ & $\delta d_{\text {con. }}$ & $\delta u_{\text {dis. }}=\delta d_{\text {dis. }}$ & & \\
\hline$\beta=5.7$, & $16^{3} \times 20$ & & & $K_{s}=0.1665$ & 0.164 & 0.160 \\
0.1665 & $0.948(31)$ & $-0.196(15)$ & $-0.044(69)$ & $-0.044(69)$ & $-0.035(58)$ & $-0.009(51)$ \\
0.164 & $0.994(11)$ & $-0.220(52)$ & $-0.027(28)$ & $-0.041(33)$ & $-0.027(28)$ & $-0.013(25)$ \\
0.160 & $1.072(50)$ & $-0.251(21)$ & $-0.008(12)$ & $-0.022(16)$ & $-0.013(14)$ & $-0.008(12)$ \\
\hline$\beta=5.7,12^{3} \times 20$ & & & $K_{s}=0.1665$ & 0.164 & 0.160 \\
0.1665 & $0.825(49)$ & $-0.227(23)$ & $0.081(84)$ & $0.081(85)$ & $0.067(74)$ & $0.053(66)$ \\
0.164 & $0.919(20)$ & $-0.242(9)$ & $0.034(38)$ & $0.035(44)$ & $0.034(38)$ & $0.026(34)$ \\
0.160 & $1.034(10)$ & $-0.257(4)$ & $0.014(17)$ & $0.016(22)$ & $0.018(19)$ & $0.014(17)$ \\
\hline \hline
\end{tabular}

TABLE II. Measured tensor charges at $\mu=1 / a$.

\begin{tabular}{|lll|ll|}
\hline \hline tensor charge & & & axial charge (ref. [11]) \\
\hline$\beta$ & 5.7 & & $\beta$ & 5.7 \\
size & $16^{3} \times 20$ & $12^{3} \times 20$ & size \\
$\delta u$ & $0.839(60)$ & $0.822(83)$ & $\Delta u$ & $16^{3} \times 20$ \\
$\delta u_{\text {con. }}$ & $0.893(22)$ & $0.760(39)$ & $\Delta u_{\text {con. }}$ & $0.638(54)$ \\
$\delta d$ & $-0.231(55)$ & $-0.159(75)$ & $\Delta d$ & $0.763(35)$ \\
$\delta d_{\text {con. }}$ & $-0.180(10)$ & $-0.220(17)$ & $\Delta d_{\text {con. }}$ & $-0.347(46)$ \\
$\delta(u, d)_{\text {dis. }}$ & $-0.054(54)$ & $0.076(71)$ & $\Delta(u, d)_{\text {dis. }}$ & $-0.226(17)$ \\
$\delta s_{\text {dis. }}$ & $-0.046(34)$ & $0.071(46)$ & $\Delta s_{\text {dis. }}$ & $-0.119(44)$ \\
$\delta \Sigma$ & $0.562(88)$ & $0.733(121)$ & $\Delta \Sigma$ & $-0.109(30)$ \\
\hline \hline
\end{tabular}

TABLE III. Tensor charges extrapolated to the chiral limit together with the axial charge obtained in ref. [1]]. The flavor singlet tensor charge is defined as $\delta \Sigma=\delta u+\delta d+\delta s$. The matrix elements are evaluated at $\mu=1 / a$. 


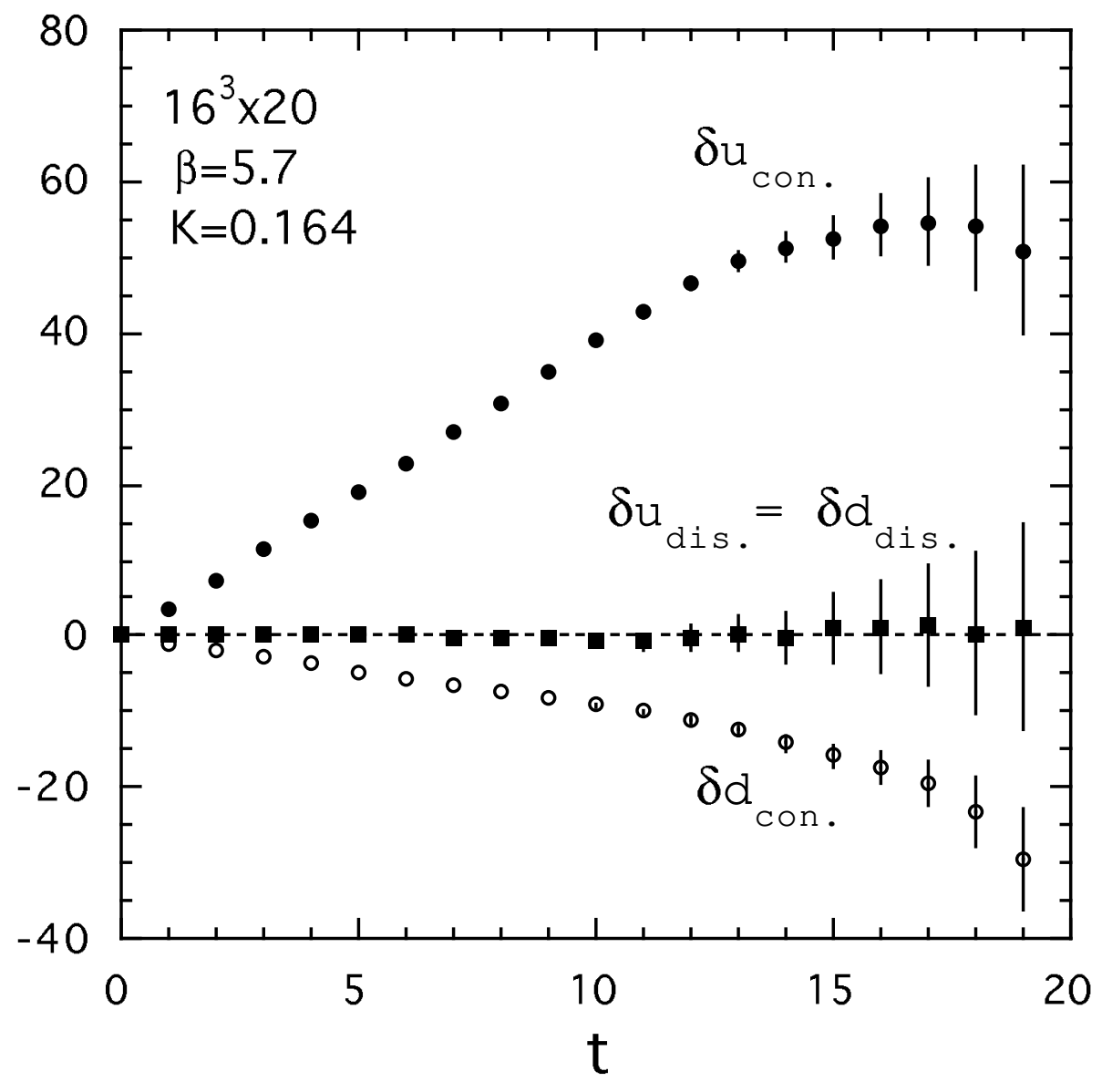




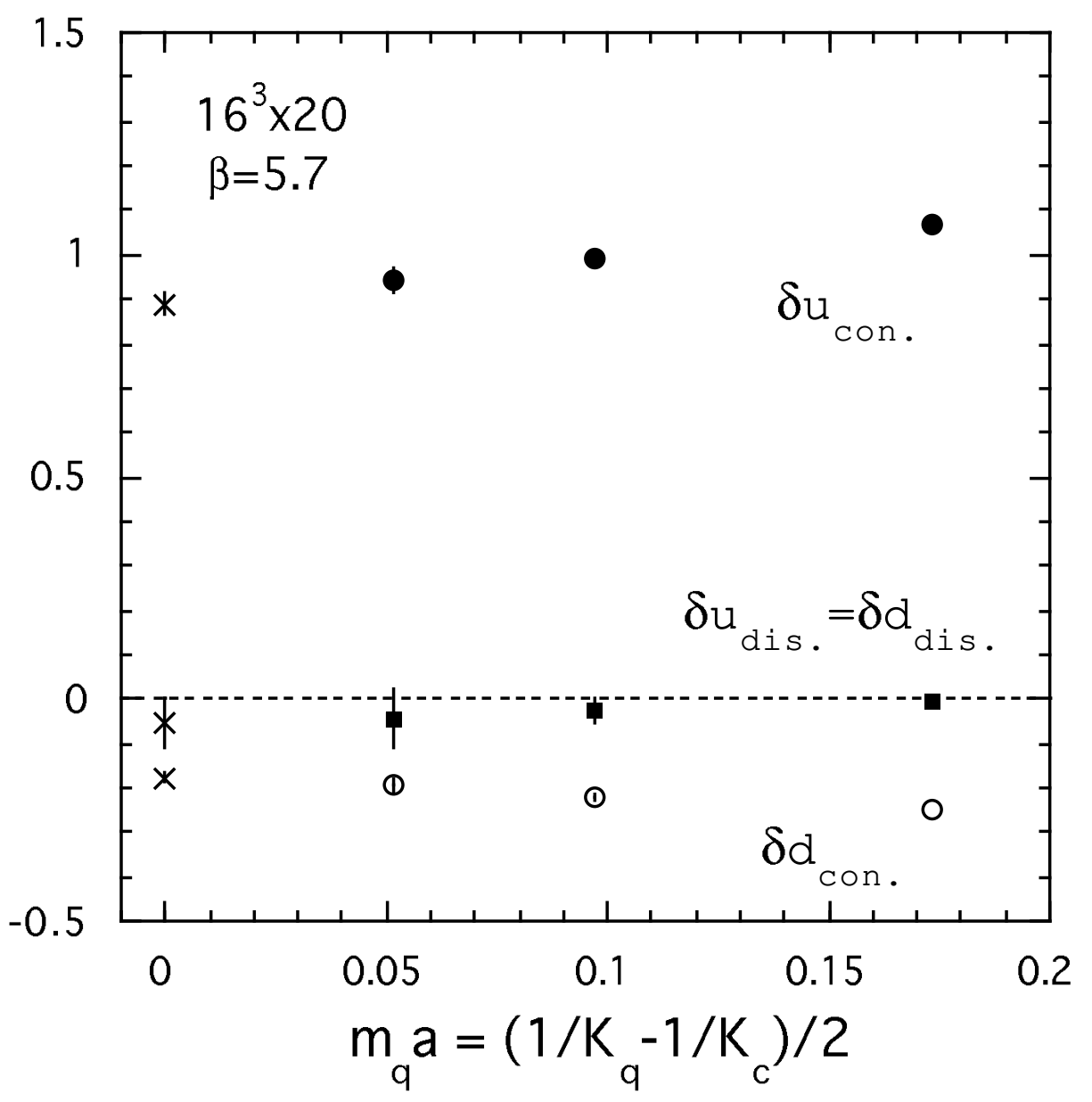

